\title{
Dynamic Distortion Correction for Endoscopy Systems with Exchangeable Optics
}

\author{
Thomas Stehle, Michael Hennes, Sebastian Gross, \\ Alexander Behrens, Jonas Wulff, Til Aach \\ Institute of Imaging \& Computer Vision, \\ RWTH Aachen University, D-52056 Aachen, Germany \\ Thomas.Stehle@lfb.rwth-aachen.de
}

\begin{abstract}
Endoscopic images are strongly affected by lens distortion caused by the use of wide angle lenses. In case of endoscopy systems with exchangeable optics, e.g. in bladder endoscopy or sinus endoscopy, the camera sensor and the optics do not form a rigid system but they can be shifted and rotated with respect to each other during an examination. This flexibility has a major impact on the location of the distortion centre as it is moved along with the optics. In this paper, we describe an algorithm for the dynamic correction of lens distortion in cystoscopy which is based on a one time calibration. For the compensation, we combine a conventional static method for distortion correction with an algorithm to detect the position and the orientation of the elliptic field of view. This enables us to estimate the position of the distortion centre according to the relative movement of camera and optics. Therewith, a distortion correction for arbitrary rotation angles and shifts becomes possible without performing static calibrations for every possible combination of shifts and angles beforehand.
\end{abstract}

\section{Introduction}

For many image processing applications like mosaicing $[1,2]$ or $3 \mathrm{D}$ reconstruction [3] the pinhole camera model is assumed as underlying imaging model. In the case of endoscopy, however, this assumption does not hold as the use of wide angle lenses leads to severe lens distortion. Many algorithms are known to compensate for this distortion, e.g. $[4,5]$.

They rely on the assumption that the optical properties of the imaging system are invariant. Systems for bladder or sinus endoscopy, however, usually feature exchangeable optics so that this assumption does not hold. Because of special mounting adapters used in those systems, which connect the optics to the actual camera, both components can be rotated with respect to each other. As the adapters exhibit some mechanical slackness both components can also be shifted with respect to each other. For these reasons, the distortion centre's location also changes, which makes a successful distortion correction using solely a conventional static approach impossible (Fig. 1). 
Fig. 1. Influence of movement of camera sensor and optics with respect to each other. Left: The elliptic field of view is located on the left of the image and its orientation marker is located at the bottom (indicated by circle). Right: The field of view is centred in the image and its orientation marker is located at the lower left. The different positions of the distortion centre in both images are indicated by the cross.
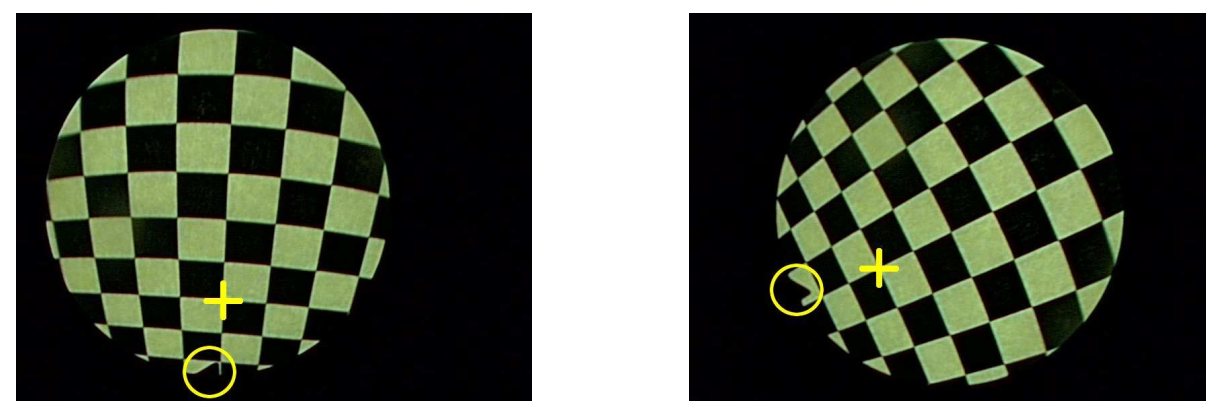

The remainder of this paper is organised as follows: We first present a method for dynamic lens distortion correction. To this end, we extend a conventional static distortion correction algorithm with the localisation and the orientation detection of the field of view in each image. Using this information, we estimate the distortion centre's new position. This, in turn, enables us to perform a distortion correction without an explicit calibration for the current rotation angle and shift. We then present a quantitative evaluation which was carried out on synthetically distorted data as well as on real calibration images acquired with an Olympus Excera II video endoscope.

\section{Materials and methods}

Our dynamic distortion correction algorithm combines the results of a static approach with the localisation and the orientation detection of the field of view (FOV, Fig. 1). The underlying assumption is that the distortion function itself does only change its position and orientation but not its actual shape when camera and optics are shifted and rotated with respect to each other.

For the initial static distortion correction, we use a planar checkerboard pattern as calibration object. The images of this pattern are analysed using Mühlich and Aach's feature extractor for high accuracy camera calibration [6]. As method for static distortion correction, we use Hartley and Kang's approach based on the fundamental matrix [5]. Since their algorithm assumes pure radial distortion, it suffices to estimate the distortion centre's new position and to shift the statically calibrated distortion function to this new location without a further change of the distortion function. However, if a distortion correction model, which also considers tangential distortion (i.e. a function which is not radially symmetric) is used, the distortion field also needs be rotated according to the FOV's rotation. 
For FOV detection, we analyse grey value profiles from the image centre to the image border pixels [7]. The position of the FOV border pixels can then be determined by finding the maximum correlation value of a step edge model and the grey value profile. As next step, an ellipse model is robustly fitted to these detected border points using the RANdom SAmple Consensus (RANSAC) algorithm [8]. The RANSAC algorithm rejects border points which do not fit the ellipse model close enough so the parameter estimation becomes more stable. The centre of the ellipse is regarded as the position of the FOV.

As the orientation marker (indicated by a circle in Fig. 1) downsizes the FOV locally, the rejected point with the largest distance to the ellipse is chosen as initial guess for the position of the orientation marker. As the region around the initial position is not sampled densely enough by the grey value profiles to allow an accurate detection of the orientation marker, the region around this point is analysed more closely in order to refine the initial guess. To this end, the contour point is found which maximises the Euclidian distance from the ellipse to the marker contour.

Finally, a new coordinate system is introduced with one axis pointing from the ellipse centre to the orientation marker. The other axis is chosen to be perpendicular to the first one. The distortion centre coordinates are now transformed to this coordinate system, which is invariant with respect to the endoscope's shift and rotation.

In our first experiment, we evaluated the repeatability of the ellipse location detection. To this end, 200 images were taken in which the location of the FOV remained constant. Subsequently, the ellipse detection was carried out and the mean distance to the location as well as the respective standard deviation was calculated.

To verify the hypothesis that the distortion centre moves along with the shift and rotation of the lens, we carried out a second experiment in which the orientation of the FOV was also taken into account. The optic was rotated to eight different angles and 200 calibration images were taken at each orientation. For each position, the distortion centre was determined using Hartley and Kang's algorithm. One of these distortion centres was defined as reference and subsequently shifted and rotated to the remaining seven positions. Then, the distance between the computed and estimated positions was calculated.

A last experiment was carried out in order to assess the impact of a slightly displaced distortion centre on the accuracy of the distortion correction. To this end, the error introduced by a distortion centre, which was displaced by the mean error found in the second experiment, was calculated using a real endoscope's distortion function.

\section{Results}

In our first experiment, we found that the mean error in the detection of the ellipse position was 0.84 pixels with a standard deviation of 0.63 pixels. Fig. 2(a) shows the results of estimating the distortion centre position. The crosses cor- 
Fig. 2. Evaluation of distortion centre estimation. (a) Position of calculated (crosses) and estimated (circles) distortion centres. (b) Mean distance between calculated and updated distortion centres with respective standard deviation.

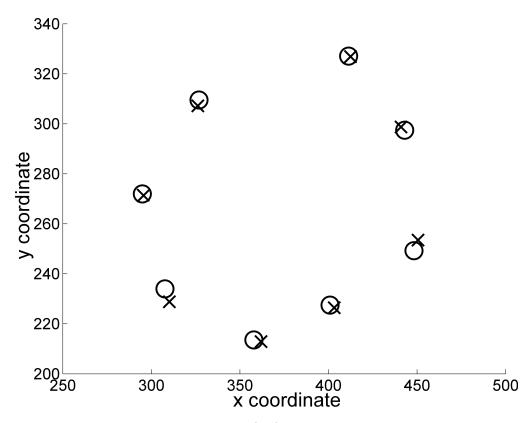

(a)

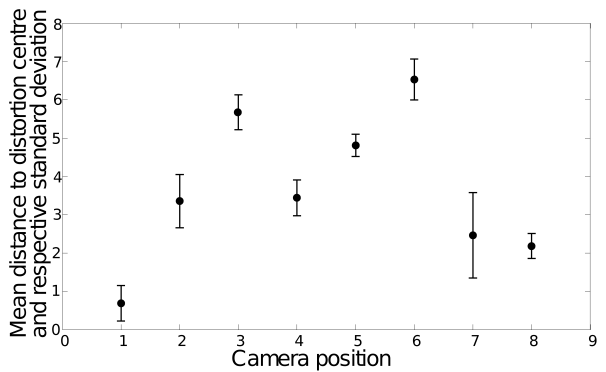

(b)

respond to distortion centres computed with Hartley and Kang's method. The circles correspond to estimated distortion centres. The mean distance between the computed and the estimated distortion centres was 3.1 pixels. These results are quantitatively shown for all rotations in Fig. 2(b).

In Fig. 3, the error in distortion corrected images introduced by a displacement of 3.1 pixels is depicted. Fig. 3(a) shows the error inside of the circular field of view. In Fig. 3(b) a profile through the error function along the plane in Fig. 3(a) can be seen. It is evident that the error in a circular region with a diameter of 450 pixels centred at the estimated centre of distortion is one pixel or lower. The whole FOV has a diameter of about 500 pixels.

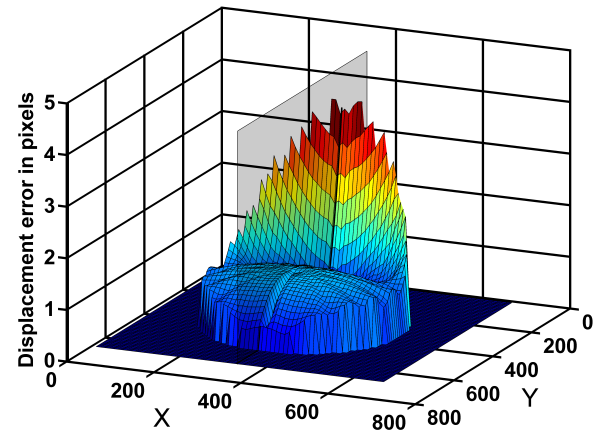

(a) $3 \mathrm{D}$ plot showing the error inside the field of view

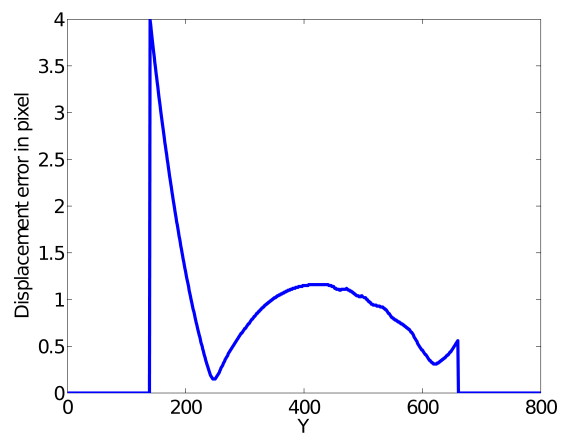

(b) Profile through 3D error plot along plane indicated in (a)

Fig. 3. Errors introduced by a distortion centre displacement of 3.1 pixels 


\section{Conclusion}

In this paper, we have identified a yet unaddressed topic in the calibration of endoscopes with exchangeable optics. These lenses cannot be fixed entirely to the camera to form a rigid object. Instead, the camera sensor and the optic can be shifted and rotated with respect to each other which has a major influence on the position of the distortion centre.

We have proposed a method to estimate the new position of the distortion centre after a rotation or a shift. Our method combines a classical approach for distortion correction with the detection of the FOV location and orientation. With our approach it becomes possible to carry out one single static calibration, and successfully compensate distortions with arbitrary rotation angle and shift of the optics with respect to the camera sensor.

In our experiments, we have shown that the proposed FOV detection method offers subpixel accuracy for the estimation of the FOV location. In a second experiment, we could verify our hypothesis that the distortion centre moves along with the FOV. In our third experiment, we have evaluated the impact of an imprecisely estimated distortion centre. As displacement we have chosen the mean error as calculated from the second experiment. We found that in a circular area around the estimated centre of distortion with 450 pixels diameter, an acceptable error of less than a pixel is present in the image.

In the future, we will investigate the sources of the remaining error. One possibility which has not yet been addressed is a tilt between camera and optics.

\section{References}

1. Konen W, Breiderhoff B, Scholz M. Real-time image mosaic for endoscopic video sequences. Proc BVM. 2007; p. 298-302.

2. Behrens A. Creating panoramic images for bladder fluorescence endoscopy. Acta Polytech: J Adv Eng. 2008;3:50-54.

3. Stehle T, Truhn D, Aach T, et al. Camera calibration for fish-eye lenses in endoscopy with an application to 3D reconstruction. Procs IEEE ISBI. 2007 April; p. 11761179 .

4. Kannala J, Brandt SS. A generic camera model and calibration method for conventional, wide-angle, and fish-eye lenses. IEEE Trans PAMI. 2006;28(8):1335-1340.

5. Hartley R, Kang SB. Parameter-free radial distortion correction with center of distortion estimation. IEEE Trans PAMI. 2007;29(8):1309-1321.

6. Mühlich M, Aach T. High accuracy feature detection for camera calibration: A multi-steerable approach. LNCS. 2007;(4713):284-293.

7. Stache NC, Zimmer H, Gedicke J, et al. Robust high-speed melt pool measurements for laser welding with sputter detection capability. LNCS. 2007;4713:476-485.

8. Fischler MA, Bolles RC. Random sample consensus: A paradigm for model fitting with applications to image analysis and automated cartography. Commun ACM. 1981 June;24(6):381-395. 